\title{
Seulement un acte : le saut du tricheur
}

\section{Christian Jouvenot}

\section{(2) OpenEdition}

\section{Journals}

Édition électronique

URL : http://journals.openedition.org/ccs/2593

DOI : $10.4000 /$ ccs. 2593

ISSN : 2558-782X

\section{Éditeur :}

Presses universitaires de Rennes, Association des lecteurs de Claude Simon

\section{Édition imprimée}

Date de publication : 30 septembre 2019

Pagination : 293-308

ISBN : $978-2-7535-7795-4$

ISSN : 1774-9425

\section{Référence électronique}

Christian Jouvenot, «Seulement un acte : le saut du tricheur », Cahiers Claude Simon [En ligne], 14 I 2019, mis en ligne le 30 septembre 2020, consulté le 10 octobre 2020. URL : http://

journals.openedition.org/ccs/2593; DOI : https://doi.org/10.4000/ccs.2593 


\title{
SEULEMENT UN ACTE : LE SAUT DU TRICHEUR
}

\author{
Christian JOUVENOT
}

«Mieux vaut tâcher d'être forte : je dois plutôt songer à construire et faire belle une jeune vie que de me lamenter sur les ruines de la mienne. Et Dieu m'y aidera. "

Lettre de Suzanne Simon à sa sœur Jeanne, 30 juin $1915^{1}$.

Le coup est terrible. La réaction immédiate. Le vécu de l'événement est aussitôt camouflé, maquillé, comme au théâtre, travesti dans un rôle. Lettre de Jeanne à son fils Louis, 9 septembre 1914: "Te dire dans quel état est ta pauvre tante [...] est impossible. Et avec cela, son calme et sa résignation chrétienne sont vraiment admirables. C'est une douleur profonde, mais résignée, bien digne de la vraie femme d'un soldat français, et qui ne peut que servir d'exemple à ceux qui la voient de près $^{2}$. " Au premier rang, c'est Claude, son fils, qui la voit de près. Il a onze mois.

Razaph, sa "mère noire " malgache, est repartie quand Claude a deux ans et demi. Elle a participé grandement à la construction du petit homme. Elle lui a offert une "alter-native ", un entre-deux mères, un espace d'échappement à l'emprise maternelle exclusive. Dans la période qui entoure la mort du père, prenant la place de la mère effondrée, elle est, pour l'enfant, au premier rang, le constant recours. C'est par là, peut-être, que Claude s'est sauvé, sa première évasion, marquée du sceau de cet attachement précoce, une marque d'une certaine façon politique : «Dans la violence qui a fendu l'âme de l'esclave, s'est ouvert

1. Citée dans F. Buffet et M. Calle-Gruber (éd.), Claude Simon, La Mémoire du roman, Lettres de son passé, 1914-1916, Les Impressions Nouvelles, 2014, p. 114.

2. Ibid. p. 103. 
un espace affectif mutilé au service du fils du maitre ${ }^{3}$. " Par la suite, Claude sera notamment soutenu par ses tantes d'Arbois qui, par leur seule présence, maintiennent l'ouverture (y compris l'ouverture politique).

Après la mort du père, après le départ de Razaph, le colloque singulier mère-fils s'est techniquement resserré dans un prêt-à-penser qui lui fait un emballage. Suzanne ne peut pas, jour et nuit, accabler l'enfant par la manifestation de son inguérissable douleur. Tricher est devenu l'ordinaire de sa passion. Comment faire autrement! Entrée dans son rôle, elle en récite le texte par cœur, celui de sa religion. Elle doit protéger son enfant en faisant bonne figure, autant qu'elle le peut, en le trompant. On le comprend sans mal. La religion lui est d'un grand secours, elle dissimule, déguise et finalement défigure la réalité profonde. C'est sous cette apparence, souvent celle d'un masque, que Claude observe sa mère, sa mère écrite dans ses romans : " le gras visage bourbonien toujours impassible derrière la trame du crêpe " ( $A$, p. 1015), devenu avec la maladie " cette momie au masque ravagé et fardé ondulée au petit fer»(Hist., p. 294).

Comme par une défense fétichique, pour tenir le coup, le prêt-à-penser est complété par un prêt-à-porter à vocation définitive. L'emballage est complet. Tout est préfabriqué, tout est faux. Ce que le fils voit de près c'est l'éteignoir du déguisement de sa mère, l'image fixe de l'affichage du drame : « les souliers noirs, les bas noirs, le manteau noir, la toque noire bordée d'un mince liséré d'où pendait le crêpe " $(A, \mathrm{p}$. 1012). Comme Molloy le dit chez Beckett, sa mère lui a donné la nuit. L'enfant, tombé dedans quand il est petit, tombé dans ce mélange de douleur et de piété, entré en danse dans ce délire à deux, feint lui aussi, inguérissable à son tour, cachant qu'il est lui aussi habité par le " désastre ", attardé dans sa solitude " au-delà de l'heure, autorisée », "irrémédiablement » enfoncé, embourbé, enlisé dans sa tragique solitude ${ }^{4}$.

Les sentiments de Suzanne doivent rester contenus, autant que possible travestis; et pourtant, dans le texte cité, elle craque, elle apparaît comme " un de ces oiseaux de basse-cour ", avec " [sa] permanente détresse, [sa] permanente panique 5 ", elle est le renversement en son contraire de la mère poule. Elle s'épuise à donner le change, tant bien que mal elle essaye de corriger le hasard, de tricher, une mascarade qui deviendra insupportable à l'enfant, « seul

3. R. L. Segato, L'Edipe Noir, Des nourrices et des mères, Petite bibliothèque Payot, 2014, p. 92.

4. C. Simon, "Sous le kimono » [1961], Cahiers Claude Simon, n 12, 2017, p. 14.

5. Ibid., p. 14. 
misérable insatisfait et vaincu ${ }^{6} "$, vaincu puisqu'il ne réussit pas à guérir sa mère, un enfant qui, lui aussi, doit faire comme si tout allait bien, jusqu'au jour où...

Tout le monde triche, peu ou prou. Rien de bizarre à ça. Ici, par exemple, à force de bricoler je me fais tricheur à mon tour; mon bricolage se construisant me séduit, retient de plus en plus mon attention; au fil du travail j'en viens à croire à ce que j'échafaude, même si je sais que l'échafaudage n'est pas le bâtiment. Que le lecteur soit prévenu, à force de bricoler je m'attache à ma construction, j'adhère à mon interprétation, je tiens mon roman qui lui ne tient qu'à un fil... C'est comme ça que je le vois, Claude Simon, le mien, " au-delà de l'heure autorisée ", seul, ayant tout abandonné, rien qu'écrire, construisant, attelé à sa tâche, petit à petit adhérant à sa phrase, sa phrase lui collant à la peau comme le sparadrap du capitaine Haddock. Mais ne voulant pas s'en défaire, y tenant, se cramponnant, "(s')attardant donc ". Son côté collage. Il adhère à son écriture pour échapper...

Retour en arrière. C'est pour échapper à la comédie du mensonge qu'il saute? Claude a moins de 10 ans. Il est à La Rochelle. Ce pourrait être dans la période de sa première communion, à 8 ou 9 ans, période également de la mort, le 19 avril 1922, de sa grand-mère maternelle : il tombe d'une hauteur de vingt mètres du haut de la tour des Quatre-Sergents. Vingt mètres! Il a une sacrée chance de s'en tirer comme ça, après un bref séjour à l'hôpital, sans plus de bobos.

La biographe accuse l'existence d'un mâchicoulis mal protégé7. Sait-on, en réalité, qui du mâchicoulis ou de l'enfant était mal protégé? Dans cette famille très catholique, l'enfant pense-t-il monter au ciel en tombant? Abandonner sa mère, la laisser sur place en abondant dans son sens, en mettant en acte son message pour rejoindre quelqu'un, frère, père, grand-mère? Rejoindre ceux qui retiennent, qui captent tout l'amour de Suzanne? Dans l'impossibilité de perdre sa mère, le suicide comme seule façon de la faire mourir en mourant avec elle? Arrêter non pas la comédie mais le manège infernal du mensonge? Retrouver Razaph? Voilà mon hypothèse : je penche pour le suicide d'un enfant. Mais lequel? Quel enfant? Claude bien sûr, mais quel Claude?

Freud, dans Psychopathologie de la vie quotidienne : "il existe, à côté du suicide conscient et intentionnel, un suicide mi-intentionnel, provoqué par

6. Ibid., p. 15.

7. M. Calle-Gruber, Claude Simon, Une vie à écrire, Le Seuil, 2011, p. 46. 
une intention inconsciente qui sait habilement utiliser une menace contre la vie et se présenter sous le masque d'un malheur accidentel ${ }^{8}$ ". Est-ce ce qui est recherché par le jeune Samuel Beckett dans un de ses jeux favoris, qui effrayait sa mère, consistant à se lancer du haut d'un grand sapin pour se sentir recueilli par ses branches inférieures ${ }^{9}$ ?

Je déroule mon hypothèse : suicide ou équivalent suicidaire, peu importe, peut-être chez Claude enfant la conséquence du sentiment de son impuissance à changer sa mère, à changer le monde autour de lui, sa conviction que rien ni personne ne peut le comprendre, le dégoût de sa trop grande passivité qui, d'un coup, est renversée en son contraire, dans sa toute puissance, sans intention vraie de se donner la mort, trouvant, inattendu, un moyen désespéré de s'individualiser, d'exister autrement...

Dix-huit ans plus tard, sur la route de Solre-le-Château, au milieu de la route des Flandres, un évènement de même nature, souvent revenu dans les romans de Claude Simon : "Et vous, jeunes gens, vous continuez? [...] [peutêtre] simplement parce que j'en étais moi aussi arrivé à agir comme un automate, je ne sais pas, je n'en sais rien, ne me demandez pas pourquoi, mais je me suis tout à coup entendu répondre : Oui, mon colonel!» (JP, p. 1108-1109).

Épisode qui retient l'auteur jusque dans son dernier livre : «il était possible de conditionner un jeune homme par ailleurs normal de telle sorte qu'il soit capable de faire montre non pas d'héroïsme, de courage, de patriotisme ou de sens du devoir mais simplement (par - mais quoi ? : abrutissement, imbécile vanité, entêtement, renoncement ou seulement insurmontable fatigue du corps et de l'esprit?) capable d'avancer, monté sur un cheval au pas, au-devant d'une mort à peu près aussi inutile que certaine» (Tram., p. 1313).

Puisque « La fin est dans le commencement [écrit Beckett] et [que] cependant on continue ${ }^{10}$ ", il peut être utile de revenir au début de la vie, au début de l'Euvre, avec, si l'on conçoit que le suicide est le meurtre d'un autre à l'intérieur de soi, cette question : qui se jette dans le vide et qui donc écrit?

C'est comme ça que j'ai relu Le Tricheur (1945) : «Ce qu'il sentait qu'il pouvait faire à l'encontre de ce qui nie et empêche, quelque chose qui les

8. S. Freud, Psychopathologie de la vie quotidienne [1922], trad. V. Jankélévitch, Payot, coll. "Petite Bibliothèque Payot ", 1967, p. 207.

9. G. Durozoi, Samuel Beckett : irremplaçable, Hermann, 2006, p. 8.

10. S. Beckett, Fin de partie [1957], Minuit, 1993, p. 91. 
étonnerait tous, simplement parce qu'il suffisait de vouloir et de jeter la raison par-dessus bord, elle et son imbécile équilibre, simplement se pencher, avoir le courage de se pencher, un tout petit peu hors des œillères de l'équilibre, le courage de... [...] Vous ne me croyez pas? dit Gauthier en relevant la tête. Je me suis laissé tomber exprès. Je me suis dit tout à coup : laisse-toi tomber! [...] Ça ne vous est jamais arrivé, dit Gauthier, d'avoir des idées comme ça on ne sait pas comment? De vous dire : si je faisais ça? [...] On a de drôles d'idées, dit-il à l'homme. Ça m'a passé comme ça par la tête de me laisser tomber"(p. 88-91). Ce qui s'appelle un passage à l'acte. Pourquoi donc se laisser tomber? Pour sortir du mensonge, pour dépasser « ce qui nie et empêche "? Pour arrêter de tricher.

Un saut dans le vide. Si le vide est vide. "Seulement un acte. Cherchant à sortir du décor, à trouver les limites du paysage coagulé, si seulement un bras dehors émergeant dans l'élément aérien " (Tr, p. 238). Pour le "remâcheur de cadavre, remâcheur de passé que je dispute au rêve " (Tr, p. 54), à la fin, la chute s'est «abymée " en rêve : "Je ne voudrais plus avoir à me tenir debout ou couché, de quelque façon que pèse mon corps. Parfois, en rêve, il m’est arrivé de tomber. Une chute sans fin, un vertige où tout se dérobait et où il n'y avait qu'à s'abandonner. Mais je ne me tuerai pas. Si seulement cette chose qui serre mon ventre et ma gorge, un flot amer de cadavres et de débris...» (Tr, p. 250 et dernière page). On a compris que j'adhère à ce propos d'Henri Godard : «Chez Claude Simon [...] l'autobiographie, avouée ou inavouée, jette rétrospectivement sur ses romans une lumière que l'on ne peut plus ignorer ${ }^{11}$. "

L'enfant a grandi. Non plus tombant, mais maintenant écrivant. Est-ce une expérience de même nature? Assurément non. Mais, je le crois, une expérience qui tente de répondre aux mêmes questions. Dans un même élan, écrire, "Seulement un acte ", emprunter, non plus la voie du passage à l'acte qui court-circuite la pensée, mais celle d'un passage par l'acte qui convoque la pensée, qui ouvre des voies nouvelles. Céline écrit la nature très laborieuse de son passage par l'acte dans une lettre à Milton Hindus : "Seulement-il y a un très grave SEULEMENT... Quand je m'approche de ces châteaux [ses romans] il faut que je les libère, les extirpe d'une sorte de gangue de brume de fatras... que je burine, pioche, creuse, déblaye toute la gangue, la sorte de coton $d u r$ 
qui les emmaillote, mirage, fouille, puis ménage [...] la transmutation du mirage au papier est pénible, lente, c'est l'alchimie ${ }^{12}$."

$\mathrm{Ne}$ plus tricher? Dur travail donc, et c'est La Corde raide! Marguerite Duras dans La Soupe aux poireaux : "On peut ne vouloir rien faire et puis, faire ça, oui, cette soupe-là : entre ces deux vouloirs, une marge très étroite, toujours la même : suicide ${ }^{13}$. " Céline, encore lui : "La création, la vraie, ça demande une grosse concentration intellectuelle, anormale, pas naturelle. J'en parle en médecin... C'est presqu'un suicide ${ }^{14}$."

Quand il écrit Le Tricheur, Claude Simon sort de sa gangue, sort d'une longue période d'errance intérieure. Qui est-il avant d'écrire? L'épisode du mâchicoulis n'a rien résolu, n'a rien changé, il souffre toujours d'une image de lui dévalorisée, d'un trouble identitaire qu'il reconnait, auquel il donne un sens, celui d'être un tricheur, un imposteur : "Et toujours dans le fond il y a la mélancolie du survivant qui accompagne le jouisseur vivant l'instant ${ }^{15}$. " Comme sa mère, il fait semblant. Il note dans ses carnets : "Faux étudiant à Cambridge, faux cavalier, faux révolutionnaire, faux guerrier-prisonnier-travail physique. Toute sa vie il a l'impression d'être resté en dehors, en retrait, spectateur, à côté, dilettante, amateur, distanciation... excitant mais pas concerné $^{16}$. "Conviction d'être dans le faux, qui, paradoxalement, signale une recherche d'authenticité, sentiment d'identité abrité pour survivre dans une identité d'emprunt. "Moi aussi, dit le narrateur de Gros-Câlin, j'aurais voulu être quelqu'un d'autre, j'aurais voulu être moi-même ${ }^{17}$. "

Tricher, pourquoi pas, c'est ruser, c'est créer déjà. Le registre du mensonge est celui d'une création, d'un roman, mais c'est une création qui pèse, une création qui a son boulet au pied tant, à la racine, elle est un mensonge partagé, quand il y a un seul mensonge pour deux qui fonctionne comme un piège. Au contraire c'est le mensonge secret, à l'insu de la mère, qui est facteur de réalisation d'une volonté propre. Gary a mis une énergie considérable à défendre son mensonge fondateur de l'existence d'Ajar. Au fond, chez Claude, continuer à tricher dans les conditions premières, celles qui pré-

12. H. Godard, Céline, Gallimard, 2011, p. 596.

13. M. Duras, Sorcières [1976], Outside, POL, 1984 p. 275.

14. Cité par H. Godard, op. cit., p. 296.

15. M. Calle-Gruber, Une vie à écrire, éd. cit., p. 325.

16. Citation des Carnets de Claude Simon, dans M. Calle-Gruber, op. cit., p. 15.

17. É. Ajar-Romain Gary, Euvres complètes, Mercure de France, coll. « Mille Pages », 1991, p. 155. 
cèdent la chute du haut de la tour des Quatre-Sergents, serait une résignation, un travestissement protecteur, une appropriation du délire à deux, une façon de ne pas grandir, de ne pas faire ses deuils, une façon de rester avec sa mère. Mais à la fin d'une adolescence qui n'en finit pas, la technique en vient à être usée, elle peine à produire ses maigres bénéfices, elle lasse. Sous ce jour, son activité de peintre est en échec, peut-être insuffisamment ancrée dans son être profond. La question déjà là dans l'enfance revient : comment s'émanciper, comment "sortir du décor ", comment s'envoler, " être quelque part où il y aurait du vent » (Tr, p. 229) ? Comment être quelqu'un?

En effet, dans la tentative de l'écriture, il y aura du Vent... Ce qui est à l'œuvre c'est "s'acharner à détruire un monde déjà mort " (Gul., p. 268), c'est trouver une ouverture plus libre que celle du mensonge partagé, c'est la voie d'une création qui délivre, celle d'un auto-engendrement : "j'imagine que les actes suffisent à s'engendrer d'eux-mêmes, il y a bien quelque chose qu'on appelle la génération spontanée ou je ne sais quoi, sans fils ni père, naissant, vivant et s'éteignant, sans avoir le temps de se regarder frapper ni mourir. L'air s'écarte quand une nouvelle personne pénètre dans une pièce, le forçant à lui faire une place " $(T r$, p. 244).

Dans Le Tricheur, qu'il s'agisse de Gauthier ou qu'il s'agisse de Louis, il faut que ça s'arrête. Le premier a essayé de se tuer, il ne se tuera pas. Le deuxième prémédite un meurtre. Louis a emprunté un revolver, il tuera, mais pas avec le révolver : il accomplira son crime en frappant sa victime avec une brique. "Alors je ramasse la brique. J'ai frappé [...] comme ça, de toutes mes forces» (Tr, p. 248).

Lourdes, combien de fois est-il allé à Lourdes avec sa mère et sa grandmère? C'est à Lourdes qu'il a fait sa communion. "Quand je me relève je comprends que c'est fini et qu'il n'y a plus rien " (Tr, p. 248). Ce geste est un geste de délivrance, il est comme un pavé dans la mare familiale, comme une claque magistrale à " la pharisienne bonne conscience ${ }^{18}$ " de sa famille maternelle, la manifestation radicale de son refus d'adhérer à la croyance de sa mère, à ce Dieu qui n'a rien fait pour elle : à Lourdes, " elle est ressortie, plus maigre encore si c'était possible, sa figure en lame de couteau, les yeux fermés, épuisés, tandis qu'alternés la voix forte du prêtre et le murmure suppliant de la foule continuaient à répondre Tour d'ivoire - Priez pour nous,

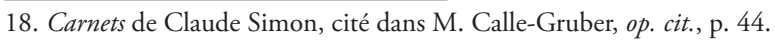


Rose de Saron - Priez pour nous - Arche sainte - Ayez pitié de nous! [...] un vertige tournoyant, harcelant, battant dans ma tête " (Tr, p. 248). Ceci trois secondes, trois lignes avant le passage par l'acte.

Claude, qui est-il devenu écrivant? "Que le travail d'écriture bloque plus que de coutume, qu'une passion l'affole, et c'est aussitôt l'impression qu'il n'y a pas d'issue, le phantasme du suicide ${ }^{19}$... " Rien n'est jamais acquis. Le mâchicoulis coule encore dans ses veines. C'est qu'il a toujours, pour toujours, parmi d'autres, un problème de taille, à régler. Peut-être celui-là : il est né après un frère mort.

À propos du suicide de Renée, sa première femme, morte le 7 octobre 1944, dans les manuscrits d'Histoire: " cette lâcheté, ce truquage qui consiste à se filouter soi-même en se dédoublant ou en essayant de se faire croire à ce double qu'on est - qu'il est - autre qu'il n'est à seule fin de s'éviter son dégoût ${ }^{20}$ ". Ce à quoi j'associe : "Mais si je me levais, la chose gluante se lèverait aussi. Moi au milieu d'eux criant et se disputant. Si je pouvais être quelque part où il y aurait du vent. Alors je m’y tiendrais debout, incliné contre lui, m'appuyant sur l'air et le sentant couler comme une eau entre mes membres et regardant le vent emporter mes vêtements au fur et à mesure que je les enlève, gonflés, s'enfuyant rapidement avec des gestes de personnages grotesques et incomplets, décroissant, trébuchant dans l'ombre et la lumière " (Tr, p. 229). On croit le voir tomber. Comment se défaire d'un double, de cette doublure, de ce fantôme, de cette chose gluante? "C'est comme si je sortais de moi, comme si j'abandonnais mon corps, la peau, la sueur qui l'enserre et son poids, comme si je devenais tout à coup invisible et présent cependant et sachant " ( $T r$, p. 242). C'est pourquoi je pose la question du suicide d'un enfant : lequel? Un suicide altruiste? Quel enfant? Claude bien sûr, " invisible et présent ", mais quel Claude, puisqu'ils ont le même prénom? Lequel des deux?

Le couple des parents, mariés le 8 février 1910, part pour Madagascar le 25 avril 1912. C'est à Madagascar que meurt leur premier enfant, entre avril 1912 et octobre 1913. Si on ne sait ni la date de la naissance, ni celle de la mort de ce premier Claude, Claude le second, né le 10 octobre 1913,

19. M. Calle-Gruber, op. cit., p. 285.

20. Ibid., p. 163. 
est né, à quelques mois près, au plus un an après la mort de son frère aîné, "prénommé Claude, déjà. Mort du croup ${ }^{21}$ ". Il est donc un enfant de remplacement, il appartient à la troupe, parmi ceux, connus, des Chateaubriand, Beethoven, Stendhal, Van Gogh et la théorie des Vincent, Camille Claudel née après un frère mort et qui fait un métier d'homme, Rilke : "j'étais tout bonnement Sophie, la petite Sophie de maman ${ }^{22}$ ", Dali : " ce jeu constant de tuer par mes excentricités la mémoire de ce frère $\operatorname{mort}^{23}$ ".

Courbet né dix-huit mois après son aîné mort à la naissance, qui se rend maître de la situation : c'est lui, Gustave, l'aîné de la fratrie, en art il est le premier, qui peint ce qui n'a jamais été peint, "Il ne faut jamais rien recommen$\operatorname{cer}^{24}$ »; de son pinceau il désigne une absence, pas de gibier, pas de trace, rien d'une existence qui l'aurait précédé, il s'invente seul face à L'Origine du Monde, au point que, prenant toute la place, "ce tableau est devenu Courbet ${ }^{25}$ ".

Duchamp, encore dans le ventre de sa mère quand celle-ci perd une petite fille de trois ans, morte du croup, Marcel qui naît six mois après la mort de Madeleine, (ce à quoi le biographe Bernard Marcadé ne consacre pas plus d'une ligne sur 500 pages, comme c'est l'habitude dans toutes ces histoires, alors qu'une vie ne suffira pas à régler l'addition), Marcel qui plus tard se travestit en RRose Sélavy, avec un air de garçon et un air de fille...

Beckett, dont la biographie, je crois, ne signale pas qu'il est un enfant de remplacement, qui lui-même n'a pas eu d'enfant, a, dans son analyse avec Bion, qui fait le lit de Compagnie, créé ce fantasme : "J'ai toujours eu la sensation qu'il y avait en moi un être assassiné. Assassiné avant ma naissance. Il me fallait retrouver cet être assassiné. Tenter de lui redonner vie ${ }^{26}$. »

Et tant d'autres, bien entendu, qui ne le savent pas toujours eux-mêmes, qui peuplent les secrets de familles...

Dans la période récente Annie Ernaux, née deux ans et demi après sa sœur morte, témoigne de sa difficulté d'être : "Entre [mes parents] et moi, maintenant, il y a toi, invisible, adorée. Je suis écartée, poussée pour te faire de la place. Repoussée dans l'ombre tandis que tu planes tout en haut dans

21. M. Calle-Gruber, ibid., p. 11.

22. R. M. Rilke, Les Cahiers de Malte Laurids Brigge [1910], Flammarion, 1995, p. 104.

23. S. Dali, L’incroyable Mr Bébé, Antenne 2, 1989.

24. G. Courbet, "Lettre aux jeunes artistes de Paris " datée du 25 décembre 1861 et publiée dans Le Courrier du dimanche du 29 décembre.

25. L. des Cars, Transferts de Courbet, Les presses du réel, 2013, p. 360.

26. F.-B. Michel, Proust et Beckett, Actes Sud, 2011, p. 80. 
la lumière éternelle. [...] Tout l'amour que je croyais recevoir était donc faux [...]. Je croyais toujours être le double d'une autre vivant dans un autre endroit. Que je ne vivais pas non plus pour de vrai, que cette vie était "l'écriture", la fiction d'une autre ${ }^{27}$."

Quant à Claude Simon : " "Il porte à sa naissance le nom d'un enfant mort. Usurpation d'identité" écrit-il le 7 mars 1981 dans ses notes personnelles, insistant sur le fait que "longtemps la pensée de ce frère dont il porte le nom" l'aura hanté. Jusque dans certains récits de rêves qu'il a transcrits et conservés $^{28}$. " Il a donc 68 ans et n'a toujours pas oublié. Pourtant bien vite la guerre, la mort du père, la mort de la mère, la mort des tantes d'Arbois, vont prendre toute la place, du moins la place manifeste..., non pas dans la vie, mais dans les romans et dans bien des travaux à eux consacrés.

Gustave, Salvador, Marcel et les autres, comme les enfants de remplacement Claude Simon a lui aussi la caractéristique de batailler pour son identité singulière au point de la vouloir incomparable, absolument originale, sans précédent, et avec eux, il a la caractéristique de ne pas avoir d'enfant, de ne pas avoir de descendance. (Courbet a bien eu un fils, mais il ne l'a pas reconnu; Beckett, dont il n'est pas avéré qu'il fasse partie de la série, n’a pas eu d'enfant.)

Claude Simon exprime lui-même, ce qui par ailleurs s'observe clairement dans son adolescence tardive, une difficulté majeure à affirmer son identité, difficulté à se séparer de l'ombre du frère mort, " ce double qu'on est ", et à vaincre la culpabilité liée. La mort de son premier enfant trop vite enfouie chez Suzanne dans la mort du beau capitaine complique encore la tâche. C'est un linceul pour deux. Tout ceci, donc, pris dans la gangue des deuils maternels.

"Pendant tout ce temps j'ai continué à sentir le malaise humide ruisselant, étroitement adapté à ma peau, comme un moule. Mais si je me lève le moule se lèvera aussi ${ }^{29}$. " Puis c'est Suzanne à son tour qui meurt. Ce sont comme des poupées russes, des moules, les morts emboîtées les unes dans les autres. On sait tout ça. Mais que penser du saut dans le vide du haut de la tour des

27. A. Ernaux, L'Autre Fille, Nil, 2011, p. 21-22, 45-46. Notons que dans La Promesse de l'aube de Gary, le fils finit par se douter que les larmes troublantes de sa mère, quand elle le regardait, ce n'était pas lui qui les inspiraient.

28. M. Calle-Gruber, op. cit., p. 11.

29. $T r$, p. 226. On peut penser aux célibataires, aux "Neufmoules mâlic " ou au «cimetière des uniformes et livrées", dans La Mariée mise à nu par ses célibataires, même de Marcel Duchamp. 
Quatre-Sergents? Du saut dans l'écriture? De ce qui s'écrit dans Le Tricheur? Qui, dans le double, tient la plume?

La chute est au début. Ce qui n'est pas sans rapport avec le fait que Claude Simon "laisse tomber"sa peinture et ses premiers romans. Tout se passe dans la perte. Et paradoxalement il accumule. En tombant, il lève les yeux, il observe le dehors avec des yeux de lynx, il observe tout dans le détail, il guette le moindre signe, la moindre étincelle dans le regard qui lui est adressé du dehors. Comme on dit "Ça me regarde ». Claude écrivant est suspendu dans le vide, avec des mots il tisse un cocon dans lequel petit à petit il se glisse, dans lequel il existe, dans lequel il habite. Il est le « ver à soie " de la dernière ligne d'Histoire.

Avec l'acharnement d'un enfant tout à la fois sans mère et qui veut être reconnu par elle, distingué par elle, il la suscite, il l'invente. Il entonne alors un chant très singulier, unique, une voix, la sienne, qui ne peut prêter à aucune équivoque. Son style est inimitable, c'est-à-dire produit dans le but ne pas être imité, si d'aventure un double cherchait encore à se glisser dans l'affaire...

Au fil des années, de Sagittaire en Calmann-Lévy, en Minuit, de Prix de l'Express en Prix Médicis, de conférences en colloques, d'honoris causa en honoris causa, de New York à Moscou, de Cerisy-la-Salle à la rue Claude Simon au hameau des Planches, du Nobel à l'édition de son œuvre dans la Pléiade, le travail d'écriture et sa reconnaissance confortent Claude, celui né le 10 octobre 1913, dans son identité propre. «Mon Claude » tient cette fois sa légitimité, sa singularité du dehors, comme un bouquet de fleurs est tenu serré par la faveur qui l'entoure. Joseph Conrad cité en exergue du Tramway: "pour lui le sens d'un épisode ne se trouve pas à l'intérieur, comme d'une noix, mais à l'extérieur, et enveloppe le conte qui l'a suscité, comme une lumière suscite une vapeur..." (Tram., p. 1251). Comme l'Euvre suscite son auteur. Claude cherche sans fin l'authentification de son être hors de lui-même. "Toute sa vie il a l'impression d'être resté en dehors. " Mais être dehors, qui pouvait être autrefois un rejet, de la même façon qu'on utilise la force de l'adversaire dans certains sports de combat, est retourné maintenant vers soi dans une manœuvre d'auto-engendrement. "Évidemment, s'agissant d'un écrivain, il faut tenir compte [...] de la présence de l'œuvre, qui, tel un 
appareil auxiliaire externe à l'appareil psychique, peut compenser les malfaçons de l'organisation intérieure ${ }^{30}$. »

Comme un saut dans le vide, courir le risque : " on efface tout et on (re) commence ". Comme Murphy chez Beckett qui se met "à voir le Rien, cet éclat incolore dont une fois sorti de la mère on jouit si rarement, et qui est l'absence ${ }^{31}$ ». Se dé-créer soi-même d'abord, un fantasme de désengendrement permettant de faire l'économie du suicide. Puis s'auto-engendrer, se vivre comme seul générateur de sa propre existence ${ }^{32}$. Artaud à propos de Van Gogh : "Car nul jusque-là n’avait comme lui fait de la terre ce linge sale, tordu de vin et de sang trempé ${ }^{33}$. " Pour Claude Simon aussi : car nul jusque-là n'avait fait comme lui. Seulement ça : nul jusque-là n'avait fait comme lui.

À propos d'Histoire, ce roman qui aurait pu s'appeler Mauvaise Foi, comme en écho au Tricheur: "Cette fois je n'avais absolument rien à "raconter" ${ }^{34}$." Au commencement, temps mort, il n'y a pas d'histoire, l'auteur commence par faire le mort. Celui qui prend la place du mort, mort à la naissance ou presque, n'a rien à raconter. C'est le silence du mort. Ceci pour vérifier que chacun est là, bien à sa place. L'autre, celui qui vient après le mort, dans un profond sentiment qu'il est à côté du mort, est comme un nouveau-né, tous les sens en éveil, pour deux. Un rien peut accaparer toute son attention : un pigeon sur un appui de pierre (1962), la branche d'un arbre contre le mur (1967), une carte postale (1973), les langues pendantes d'un papier peint (1975), un dessin à la mine de plomb (1981), le tableau de bord d'un tramway (2001). Puis l'ampleur de la tâche, le côté Cézanne de La SainteVictoire, les reprises, remaniements à n'en plus finir, compositions, montages; offerte comme l'image de soi dans le regard de la mère, l'écriture comme un présent, la phrase surtout, "en dehors " et revenue du dehors, la nature sans précédent de la phrase, si fragmentée et agrégée, si composite et à la fois si tenue, son foisonnement, son amplitude démesurée, sa dérive, son odyssée, sa fonction de labyrinthe (Icare en haut de la tour des Quatre-Sergents), sa capacité d'enchantement, sa fonction Shéhérazade qui intime de ne pas

30. A. Bauduin, Psychanalyse de l'imposture, PUF, 2007, p. 73.

31. S. Beckett, Murphy, Minuit, 1951, p. 177.

32. P. C. Racamier, Cortège conceptuel, Apsygée éditions, 1993.

33. A. Artaud, Van Gogh, le suicidé de la société [1947], Gallimard, coll. "L'Imaginaire », 1990, p. 44.

34. P. Descargues, La Tribune de Lausanne, 1967, cité dans E II, p. 1396. 
lâcher sous peine de mort, qui tient éveillé, qui tient le bonhomme... les fils savamment enchevêtrés d'un tissage, un enveloppement, jamais le même, un cocon toujours recommencé.

Commencé fin 1963, Histoire se termine fin 1966 sur une question : " ce sein qui déjà peut-être me portait dans son ténébreux tabernacle sorte de têtard gélatineux lové sur lui-même avec ses deux énormes yeux sa tête de ver à soie sa bouche sans dents son front cartilagineux d'insecte, moi ?..." (Hist., p. 416). Dernier mot d'Histoire : "moi?... "C'est lui ou c'est moi?, avec ce point d'interrogation resté à la fin en suspension. Qui est ce têtard? Qui est moi? Quel moi ? Comme le leitmotiv à la fin de La Route des Flandres: "mais comment savoir, comment savoir?" $(R F$, p. 408). Depuis vingt ans le tricheur a grandi, depuis vingt ans il s'interroge. Existentiel "qui suis-je? ", passion ordinaire, mais ici parasitée par Claude est/et Claude, insecte dans la tête, c'est-à-dire segmenté, coupé en deux, condamné à perpétuité à exister en morceaux.

Bien sûr, en ouverture d'Histoire, le contenu de l'exergue a grand sens au regard de l'aventure de l'écriture et de la composition du roman. Par affinité élective, faire le choix de Rilke, "la petite Sophie de maman ", anticipe le questionnement du dernier mot : "Cela nous submerge. Nous l'organisons. Cela tombe en morceaux. Nous l'organisons de nouveau et tombons nousmêmes en morceaux " (Hist., p. 145). Retour du mâchicoulis. On n'en finit pas de tomber! Avec ça, ramasser les morceaux, ce qu'il fabrique en lui pour/ contre sa mère, c'est René avec/après Sophie, morte un an avant sa naissance. Rainer, Renée Clog, sa première femme, suicidée en octobre 1944. "Nousmêmes ", Claude/Re-né, moi, moi?...

Des morceaux. Ce tas de gravats au pied du mur dans la maison de Leçon de choses : " morceaux de bois, de brique " (LC, p. 557). Dé-créer d'abord, puis "EXPANSION ", la construction du monde commencée, à " petits coups de pinceau ». Dans Le Tricheur, une brique. Est-ce seulement anecdotique? Au contraire, c'est tout un symbole. Louis n'utilise pas le revolver qu'il a emprunté à Armand. Armand armant Louis, une forme de complicité, antérieure aux faits, d'avant la naissance de l'acte, un retour du double contraire à l'affirmation de soi en tant qu'identité une, indivisible, et surtout sans précédent. Non. Le geste de Louis ne doit rien devoir à personne d'autre 
qu'à lui-même. Une situation tout autre que celle de L'Étranger : Meursault sur la plage avec le pistolet de Raymond.

"Et je m'arrête comme pour pisser contre le tas de briques tandis qu'il continue tranquille le long de l'échafaudage. Alors je ramasse la brique " (Tr, p. 248). C'est l'endroit d'un chantier : un tas de briques, des sacs de ciment, une " odeur de plâtre frais", des planches, une palissade. Le chantier du roman. Une brique, l'arme du crime, l'instrument du geste libérateur. Des briques en alternance avec des galets, une Page d'écriture telle qu'elle est photographiée par Claude Simon (Phot., p. 86). C'est avec une brique qu'il règle son compte avec ses fantômes, qu'il démolit l'ancien monde. C'est avec une brique, dans cette neuve solitude, que dans Le Tricheur il commence la construction, commencement de l'CEuvre. À partir de là, selon l'expression de Ricœur, il continue à commencer, travaillant à « des Renaissances inattendues ${ }^{35}$ ".

P.-S.

Le processus de désengendrement et d'auto-engendrement évoqué dans ces lignes, mis en œuvre à partir de l'écriture du Tricheur, ne cesse plus, dès lors, de participer à l'aventure de l'écriture, comme on peut le lire dans L'Acacia (p. 1033) : " en passant par une succession de phases au cours desquelles ils auraient d'abord soudainement grandi, atteint à toute vitesse le stade de la puberté (l'évolution commençant à la première attaque d'avions), puis d'hommes faits (l'unique jour où ils avaient pu se battre), puis [...] défaits, dans tous les sens du terme, [...] mais encore en tant qu'individus : comme ces paquets, ces sacs dont sitôt le cordon qui les lie dénoué ou tranché le contenu se répand, roule et s'éparpille dans toutes les directions ", etc. Je veux souligner ici que ce processus trouve un renfort durablement déstructurant-structurant dans le vécu de l'expérience de la guerre. Ainsi chez l'écrivain la phrase bataille, déstructurée-structurée. Sans le vécu de la guerre et du Stalag, expérience de mort et de renaissance répétant à grande échelle le mâchicoulis personnel, chez Claude Simon, dionysiaque, l'acte d'écrire aurait

35. "J'attends la Renaissance », entretien avec Paul Ricœur, réalisé et publié par Joël Roman et Étienne Tassin, dans J. Message, J. Roman et E. Tassin (éd.), À quoi pensent les philosophes?, Autrement, $\mathrm{n}^{\circ} 102$, 1988 [http://www.fondsricoeur.fr/uploads/medias/articles_pr/attends-la-renaissance.pdf]. 
peut-être épuisé cette tentative de se recréer, comme celle-ci s'est auparavant épuisée dans l'acte de peindre.

Lisant à l'instant ces mots écrits du front en 1916 par Jean-Richard Bloch dans une lettre adressée à Romain Rolland ${ }^{36}$ : «J'exprimerai un jour, si je le peux, cette volupté rare de n'être plus qu'un atome au cœur d'un peuple entier, une poitrine parmi d'autres poitrines, un combattant nu et sans protection, au coude à coude d'un million de frères semblables à vous. " Je trouve magnifiquement exprimé le fruit de ce mouvement complexe de régression, de cette active passivité, de régression et d'expansion, qui crée une porosité identitaire, ce que j'ai désigné par «le métissage du personnel et du collectif [...] qui vient soutenir et nourrir de façon essentielle la quête identificatoire ${ }^{37}$ ». Un mouvement qui dénonce, sinon la vanité, du moins la relativité d'une identité " armée ", comme on le dit du béton, d'une identité toute personnelle, tout à soi, pointant l'obstacle qu' elle constitue quand elle est un refuge, lorsqu'elle résiste, qu'elle se crispe ou se fige, comme un Moi « embastillé » (Michel de M'Uzan), sur le chemin d'une rencontre de l'homme avec lui-même. Et plus il y a d'insécurité intérieure, plus le cramponnement identitaire est puissant.

Apparent paradoxe que cette "volupté rare de n'être plus qu'un atome ", fleur enracinée dans la boue des tranchées, cueillie près de la mort, près des corps à moitié ensevelis ou accrochés aux barbelés. Dans la guerre, l'hypothalamus commande par vagues des montées d'adrénaline. Un certain dérèglement des sens «fait son remuement dans les profondeurs» (Rimbaud dans sa lettre à Paul Demeny dite "du voyant »). Pour Claude Simon : "Essayez de vous chercher. "Je est un autre". Pas vrai : "Je est d'autres". D'autres choses, d'autres odeurs, d'autres sons, d'autres personnes, d'autres lieux, d'autres temps " $(C R$, p. 174). La présence "d'autres personnes ", la présence des fantômes de la vie personnelle est accueillie, avalée, absorbée par/dans la présence "d'un million de frères semblables ", noyant par/dans le nombre l'angoisse identitaire de l'enfant de remplacement. "Je est d'autres. " Beckett écrit dans L'Innommable: "Je ne dirai plus moi. Je ne le dirai plus jamais, c'est trop bête. " Dans le nombre des combattants qui peut savoir s'il est bientôt le remplacé ou le remplaçant d'un frère semblable?

36. J. Guéhenno, La Mort des autres, Grasset, 1968, p. 146.

37. C. Jouvenot, op. cit., p. 214-215. 
Jusque-là inconnue, soudain délivrée, une émotion puissante au plus profond a balayé le sentiment d'une identité jalouse repliée sur elle-même. Une "volupté rare " chez Jean-Richard Bloch. La même expérience chez Claude Simon, "une tendresse violente et absurde " dont il témoigne dans La Corde raide (p. 50) : "La vie que j'allais perdre infiniment adorable, soudain infiniment familière et douce, la vie à laquelle déjà je n'appartenais plus, se personnifia d'abord dans une foule impersonnelle, les cavaliers de mon escadron, une tendresse violente et absurde pour tous ces hommes envers lesquels je ne ressentais pourtant qu'une sympathie modérée. "

C'est dans cette forme de la rencontre que s'enracine profondément l'empathie dont Claude Simon est remercié lors de la réception de son prix Nobel.

Écrivain, psychanalyste et psychiatre, Christian Jouvenot est notamment l'auteur de Claude Simon. L'identification au père inconnu (L'Harmattan, 2015). 